\title{
Relación de la motivación, intención de ser físicamente activo y satisfacción deportiva en Educación Física
}

\section{Relationship of motivation, intention to be physically active and sports satisfaction in Physical Education}

\author{
Lorena Elisabeth Basurto-Ortiz \\ lorena.basurto.77@est.ucacue.edu.ec \\ Universidad Católica de Cuenca, Azogues \\ Ecuador \\ https://orcid.org/0000-0001-5131-7313 \\ Diego Andrés Heredia-León \\ diego.heredia@ucacue.edu.ec \\ Universidad Católica de Cuenca, Cuenca \\ Ecuador \\ https://orcid.org/0000-0003-2671-8961
}

Recepción: 30 de agosto 2021

Revisado: 20 de septiembre 2021

Aprobación: 15 de noviembre 2021

Publicación: 01 de diciembre 2021 
Revista Arbitrada Interdisciplinaria KOINONIA

Año VI. Vol VI. N4. Edición Especial: Educación III. 2021

Hecho el depósito de Ley: FA2016000010

ISSN: 2542-3088

FUNDACIÓN KOINONIA (F.K). Santa Ana de Coro. Venezuela.

Lorena Elisabeth Basurto-Ortiz; Diego Andrés Heredia-León

\title{
RESUMEN
}

El objetivo del estudio fue determinar la correlación que existe entre la motivación, la intención de ser físicamente activo y la satisfacción intrínseca en el deporte en estudiantes de Educación Física. Se realizó un diseño transversal descriptivo mediante correlaciones bivariadas, con una población de 1035 estudiantes, 508 hombres y 527 mujeres, con una edad media $(M=13.24$; $D S=2.61)$. Se utilizó el cuestionario de regulación de conducta en el deporte; cuestionario de medida de la intencionalidad para ser físicamente activo y cuestionario de satisfacción intrínseca en el deporte. Los resultados destacan la relación entre la Motivación con la Intención de ser Físicamente Activo y la Satisfacción Intrínseca de Diversión. En conclusión, se demuestra la importancia de que los docentes impulsen la motivación, lo cual desarrolla la intención de ser físicamente activo y la diversión, siendo una secuencia de adherencia a las clases de Educación Física.

Descriptores: Motivación; educación física; deporte. (Palabras tomadas del Tesauro UNESCO).

\begin{abstract}
The objective of the study was to determine the correlation that exists between Motivation, the Intention to be Physically Active and Intrinsic Satisfaction in Sport in Physical Education students. A descriptive cross-sectional design was carried out using bivariate correlations, with a population of 1035 students, 508 men and 527 women, with an age $(\mathrm{M}=13.24$; $\mathrm{DS}=2.61)$ from the Fiscomisional Educational Unit "Don Bosco" of Macas. For the collection of the information, the Questionnaire of Regulation of Conduct in Sport was used; Questionnaire for Measuring the Intent to be Physically Active and Questionnaire for Intrinsic Satisfaction in Sport. The results determined the relationship between General Intrinsic Motivation, MI of Knowledge, MI of Stimulation, MI of Achievement, Integrated R, Identified R, Introjected R, External R and Demotivation; The Intention to be Physically Active and the Intrinsic Satisfaction of Fun and Boredom in Sports in Physical Education students. In conclusion, the importance of teachers promoting motivation in their classes is demonstrated, which develops the intention of being physically active and having fun in the classes, a sequence of adherence to Physical Education classes.
\end{abstract}

Descriptors: Motivation; physical education; sport. (Words taken from the UNESCO Thesaurus). 


\section{INTRODUCCIÓN}

Las clases de Educación Física (EF) ayudan en el progreso e impulso de la Actividad Física (AF) entre los estudiantes, creando hábitos de práctica que duren toda la vida. Numerosos estudios como los de (Cera-Castillo et al. 2015), manifiestan que los ambientes educativos y la motivación de niños y adolescentes en las clases de EF intervienen en el desarrollo de la intención de estar físicamente activo con actitudes vinculadas en esta actividad. Es fundamental saber qué aspectos pueden mejorar la motivación, intención y satisfacción en el deporte, en estudiantes de EF con miras a mejorar las prácticas escolares de AF.

En este sentido, las instituciones y los sistemas educativos siguen anclados en formas tradicionales de enseñanza. La mayoría de los jóvenes se cansan, les falta motivación para seguir aprendiendo, en ocasiones reprimen sus talentos. Así pues, el trabajo que se presenta puede ayudar a mejorar algunos de los problemas que enfrentan los maestros actualmente, puede proporcionar información de valiosa, incluidos algunos problemas de comportamiento que enfrentan los estudiantes (Manzano-Sánchez \& Valero-Valenzuela, 2013).

Cada vez son más preocupantes los niveles de inactividad física en los niños y adolescentes, siendo uno de los diez principales factores de riesgo y de muerte en todo el mundo y responsable de enfermedades significativas según la (Organización Mundial de la Salud, 2020). En la actualidad, la inactividad física es la causa que se desarrollen graves consecuencias para el bienestar de las personas en relación con la salud (Garcés, et al. 2015). Dado que la EF es obligatoria en el currículo educativo y que la mayoría de los alumnos la siguen durante las distintas etapas escolares, esta asignatura se ha sido considerado el eslabón adecuado para promover estilos de vida saludables. Sin embargo, corresponde a los docentes de EF incrementar la participación y motivación de los jóvenes, para que se propongan participar en prácticas deportivas extraescolares (Méndez-Giménez et al., 2013; Moreno-Murcia et al. 2019). 
La motivación es un aspecto de gran importancia en el área de la educación, por cuanto orienta los trabajos y se conforma como mecanismo central que realiza un individuo y hacia qué objetivos se dirige. La teoría se enfoca en los tipos, más que en la cantidad, de motivación, prestando especial atención a la automotivación, la motivación controlada y la desmotivación como predictores de resultados de desempeño, relación y bienestar, (Deci \& Ryan, 2008). Además, la motivación se entiende como un aspecto clave de cualquier actividad que pueda realizar una persona, ya sea en el ámbito laboral, en la ciencia o en el deporte, es decir, que los resultados tienen que ver con la motivación de las personas en ese momento de realizar la actividad (Mendelsohn, 2001). Así mismo, se logra definir a la motivación como desarrollar que la persona se fije un objetivo, gestione los recursos adecuados y mantenga un comportamiento determinado, con el objetivo de lograr una meta (Naranjo, 2009).

Por un lado, la motivación en clases de EF es una de las razones importantes para analizar la incidencia en el aprendizaje. Por esta razón, (Rodríguez, 2009), manifiesta que, en el ámbito educativo, la motivación debe reconocerse como una capacidad positiva para aprender y crear de forma autónoma. Con esta definición, es evidente la importancia de la motivar a los estudiantes de EF para que logren resultados positivos. No obstante, la mayoría de las investigaciones se han centrado en seguir la línea de la motivación del deportista (Franco et al. 2017) que han acentuado la importancia de analizar, además de las variables motivacionales, los métodos cognitivos que contribuyen a mejorar el bienestar y la satisfacción del entorno de la AF. Incluso, esta motivación positiva acarrea una serie de resultados significativos, como el empeño de realizar una práctica deportiva (Lim \& Wang, 2009; Sproule et al. 2007) y la alegría del deportista (Moreno et al. 2008). Así pues, la motivación es un punto clave para lograr comprometerse y adherirse al deporte, por ser importante en el comportamiento humano (Moreno et al. 2007).

De esta manera, la motivación se ha estudiado mediante la Teoría de la Autodeterminación (TAD) de (Ryan \& Deci, 2000), que ha sido dividida en varios niveles 
como es la Motivación Intrínseca (MI), Motivación Extrínseca (ME) y Desmotivación o Amotivación (A). Así pues, la MI implica realizar actividades por el placer derivado de su realización, siendo innecesarias las recompensas externas. Además, (Méndez-Giménez et al., 2013), manifiesta que la motivación intrínseca conduce distintos tipos de modos adaptativos de carácter cognitivo, emocional y conductual para su rendimiento y satisfacción; Asimismo (Charchaoui et al. 2017; Menéndez \& Fernández-Río, 2017); hacen referencia al hecho de realizar cualquier actividad por propio placer e interés, que se obtiene en el desarrollo de la AF. Profundizando más, la TAD de (Ryan \& Deci, 2000), se manifiesta en tres tipos de motivadores intrínsecos, la $\mathrm{Ml}$ de conocimiento es cuando se practica deporte por el placer de saber más; la MI de estimulación es la que se practica por vivir experiencias placenteras; la MI de consecución hacer deporte para mejorar sus habilidades, por el contrario, siguiendo a (Ryan \& Deci, 2000), la SE ME refiere a la concepción de la actividad como un medio para lograr algo, de este modo, los estudios de (Carrasco et al. 2015; Fernándes \& Silveira, 2012), manifiestan que la motivación extrínseca es aquella que proviene de agentes externos y es determinada por recompensas que funciona como un motor para poder realizar algo, y a la vez se manifiestan en diferentes grados: regulación integrada que implica no solo identificarse con la importancia de las conductas, sino integrarse de forma armónica y coherente con otras situaciones, como valores, metas, necesidades personales e identidad; en cambio, la regulación Identificada se valoran los beneficios del ejercicio físico y la importancia que tiene su práctica.

Por otra parte, la regulación introyectada son conductas que reflejan el sentimiento de culpa y obligación que caracteriza el deseo de practicar; sin embargo, en la regulación externa las conductas son controladas por fuentes externas, para obtener incentivos externos u obligaciones impuestas por otros; por último, la desmotivación hace referencia a la falta de intención o ausencia de motivación (Moreno-Murcia et al. 2014), es decir, la desmotivación refleja una falta de intención de participar en el comportamiento. Referente a todo esto, las mencionadas variables han sido estudiadas en el ámbito de 
la EF por (Cera-Castillo et al., 2015; Espinoza-Arias \& Heredia-León, 2021; Usán \& Salavera, 2018).

También estas variables en el ámbito del deporte coinciden con resultados de otras investigaciones y han sido mencionadas en estudios como (Almagro et al. 2011; Balaguer et al. 2008). En la variable de la Intención de ser físicamente activo se indica el propósito expresado por los niños de practicar AF puede ser un buen indicador de la motivación para adquirir la actividad, así como un indicador del procedimiento futuro (Shephard \& Trudeau, 2000). A partir de los estándares de aprendizaje en la materia de EF durante la etapa de la secundaria, la práctica habitual de AF y condición física, que asocia el efecto de esta práctica con la mejora de la calidad de vida, se consiguen resultados positivos (Moreno-Murcia et al., 2019) como la intención de ser físicamente activo (Sánchez-Oliva et al. 2012). Es decir, es descrita como calcular el grado de motivación de las personas, para ser físicamente activo tras el paso por los establecimientos educativos, más allá de las clases de EF, ha sido estudiada por varios autores tanto en estudiantes de EF, (Franco et al. 2016; Moreno et al. 2012; Munuera et al. 2018). Así como en el ámbito deportivo (Almagro \& Conde, 2012; Franco et al. 2012; Valero-Valenzuela et al. 2019).

La Satisfacción Intrínseca en el deporte nos menciona que a más de la motivación, (Baena-Extremera \& Granero-Gallegos, 2014) también confirman que la satisfacción / diversión y aburrimiento que presenta el alumno en relación al deporte puede ser coherente con el interés por la EF y la importancia que tiene en adquirir hábitos de actividad física. Diversos estudios han demostrado que la satisfacción y diversión de (Baena-Extremera \& Granero-Gallegos, 2015; Granero-Gallegos et al. 2014; Zueck, Ramírez et al. 2020), experimentan los estudiantes en clases de EF son determinantes para su futuro estilo de vida. Además, en la satisfacción en el deporte es posibilitar a los niños y jóvenes una participación divertida, una práctica donde se incremente la motivación, las ganas de participar y determinantes del interés de los estudiantes por la disciplina deportiva como resultado de la adquisición y adherencia de hábitos de AF 
(Franco et al., 2016), estudios relacionados en el ámbito deportivo encontramos en (Abraldes et al. 2016; Baños et al. 2019; Gutiérrez-García et al. 2020).

Efectivamente, diversos estudios (Méndez-Giménez et al. 2012), mostraron la conexión entre la motivación de la EF y los diversos efectos: el esfuerzo en las clases de EF e intención de ser físicamente activo fuera de la institución educativa, disfrute de los deportes (González-Cutre et al. 2008) y mayor tiempo de práctica deportiva extraescolar (Almagro et al. 2012; Moreno et al. 2009). No obstante, a nivel escolar se observa un índice significativo de niños con un estilo de vida activo, índice que disminuye aún más durante el bachillerato y la edad adulta (Garcés et al. 2015). Por consiguiente, en EF un maestro que ofrece opciones de comportamientos y tareas está fomentando la autonomía, reduce las presiones de control para comportamiento uniforme y mejora la motivación (Lim \& Wang, 2009).

Por todo lo antes mencionado, como objetivo de esta investigación ha sido conocer la influencia de la motivación sobre la intención de ser físicamente activo y satisfacción deportiva en estudiantes de EF a través de análisis descriptivos y correlaciones para verificar su predominio en función de la edad, género, grado o curso de los estudiantes.

\section{MÉTODO}

El diseño del presente estudio corresponde a un enfoque de investigación cuantitativo, descriptivo de corte transversal, se sigue la sugerencia de (Montero \& León, 2007) porque recopilan datos en un sólo instante, en un solo tiempo. Es decir, su propósito se centra en la descripción de variables y el análisis de su comportamiento en un momento dado.

\section{Participantes}

La población objeto de esta investigación está conformada por 1035 estudiantes, 508 hombres y 527 mujeres, en edades consideradas entre los 8 y 22 años de edad ( $M=13.24$; $D S=2.61)$, son estudiantes de la Educación Básica Media, Básica Superior y 
Bachillerato General Unificado de la Unidad Educativa Fiscomisional "Don Bosco" Macas, en el período académico 2020-2021, los estudiantes de la institución llevan practicando deporte extraescolar una media de $(M=1.45 ; D S=.49)$, de las cuales se dedican a entrenar a la semana una media de $(M=2.67 ; D S=2.44)$, ocupando horas a la semana practicando deporte una media de $(M=1.16 ; D S=1.20)$ y finalizando con sesiones de entrenamiento con una media de $(M=.82 ; D S=.79)$. Se aplicó un muestreo que corresponde al intencional por accesibilidad.

\section{Instrumentos}

Cuestionario de Regulación de Conducta en el Deporte (BRSQ), (Lonsdale et al. 2008), el cuestionario está compuesto por 36 elementos divididos en 9 escalas que miden $\mathrm{Ml}$ general, Ml conocimiento, Ml estimulación, Ml consecución, R integrada, R identificada, $R$ introyectada, $R$ externa y Desmotivación todas estas variables se componen de 4 elementos. Las respuestas se recopilan en una escala tipo Likert de 5 puntos que van desde nada es verdad (1) a muy verdadero (5). La escala evidencia valores Alpha de 0.88 para la motivación intrínseca general, para la motivación intrínseca hacia el conocimiento 0.91, para la motivación intrínseca hacia la estimulación 0.90 , para la motivación intrínseca hacia la consecución 0.90 , para la motivación extrínseca integrada 0.91, para la motivación extrínseca identificada 0.89 para la motivación extrínseca introyectada 0.80 , para la motivación extrínseca externa 0.85 , para la desmotivación 0.86 .

El cuestionario de Medida de la Intencionalidad para ser Físicamente Activo de la versión adecuada al contexto mexicano (MIFA) (Moreno et al., 2007), que tiene cinco elementos que pertenecen para medir la intención del joven de ser físicamente activo luego de haber completado varios años de básica y de bachillerato de la institución educativa. Para los ítems que fueron precedidos por la oración "Con respecto a su intención de realizar ejercicio físico...", los participantes respondieron en una escala Likert de 5 puntos, que van desde totalmente en desacuerdo (1) hasta totalmente de acuerdo (5). La escala 
muestra una valoración Alpha de 0.81 para la medida de la intencionalidad para ser físicamente activo.

La versión española del Cuestionario de Satisfacción Intrínseca en el Deporte (SSI), (Balaguer et al. 1997; Castillo et al. 2002; Duda \& Nicholls, 1992), el cuestionario consta de 8 elementos divididos en dos escalas que miden la Satisfacción / Diversión con 6 elementos y el Aburrimiento con 2 elementos que indican criterios de diversión y aburrimiento. Las respuestas se almacenan en una escala tipo Likert de 5 opciones que van desde muy en desacuerdo (1) a muy de acuerdo (5). La escala indica una valoración Alpha de 0.88 para la satisfacción intrínseca hacia la diversión, y para el aburrimiento muestra 0.86 .

\section{Procedimiento}

Se contactó con la autoridad del plantel para solicitar el permiso necesario para aplicar los instrumentos, además, se realizó una reunión con los docentes de Educación de la institución para informales la finalidad de la investigación y para pedir la colaboración de que los alumnos llenen un cuestionario en los encuentros virtuales. Los cuestionarios se presentaron en la mayoría en las clases de EF. Bajo el control del investigador se llevó la realización del cuestionario, de esta manera se podía solventar cualquier duda que se diera durante el cumplimiento de este proceso. Se informó el anonimato de la información para que las respuestas sean sinceras, el tiempo notificado para la contestación fue aproximadamente de 20 minutos.

\section{Análisis Estadístico}

Se realizó análisis a las variables sociodemográficas, de frecuencias y análisis estadístico descriptivos, correlaciones bivariadas y de fiabilidad, se utilizó el programa SPSS v24.0. 


\section{RESULTADOS}

\section{Variables demográficas}

Tabla 1.

Datos sociodemográficos y deportivos.

\begin{tabular}{lccc}
\hline Edad del Estudiante & Frecuencia & Porcentaje & Porcentaje válido \\
\hline 8 a 11 años & 316 & $30.53 \%$ & $30.53 \%$ \\
12 a 19 años & 719 & $69.47 \%$ & $69.47 \%$ \\
Total & 1035 & $100 \%$ & $100 \%$ \\
\hline
\end{tabular}

\begin{tabular}{lccc}
\hline Subnivel y nivel educativo & Frecuencia & Porcentaje & Porcentaje válido \\
EGB Media y Superior & 705 & $68.12 \%$ & $68.12 \%$ \\
BGU & 330 & $31.88 \%$ & $31.88 \%$ \\
Total & 1035 & $100 \%$ & $100 \%$ \\
\hline & & & \\
\hline Datos deportivos & Rango & Media & Desviación Estándar \\
Deporte extraescolar & $1(\mathrm{Si}) 2(\mathrm{No})$ & 1.45 & .49 \\
Días de entrenamiento a la semana & $1-7$ & 2.67 & 2.44 \\
Horas al día de entrenamiento & $1-5$ & 1.16 & 1.20 \\
Sesiones al día de entrenamiento & $1-3$ & .82 & .79 \\
\hline
\end{tabular}

Nota: EGB = Educación General Básica, BGU= Bachillerato General Unificado

Fuente: Programa estadístico SPSS v24.

En la Tabla 1, se observa los datos socio demográficos y deportivos de los estudiantes de la Unidad Educativa Fiscomisional "Don Bosco" de Macas, que participaron en las encuestas, estableciendo una serie de variables demográficas: en cuanto a la edad el $69.47 \%$ corresponden a jóvenes entre los 12 a 19 años de edad, mientras que el $30.53 \%$ son infantes con edades entre 8 a 11 años; siendo el género de los estudiantes femeninos y masculinos; refiriéndose al subnivel de Educación General Básica Media (5tos, 6tos y 7 mos grados) tiene un porcentaje mayor de $68.12 \%$ de estudiantes, 
mientras que en el nivel educativo de Bachillerato General Unificado (1eros, 2dos y 3eros cursos) cuenta con un porcentaje de $31.88 \%$; finalmente, en lo referente a lo deportivo la media de estudiantes que practican deporte extraescolar es bajo (1.45); los días de entrenamiento a la semana es muy poco (2.67); las horas de entrenamiento (1.16) y por ultimo las sesiones de entrenamiento muy escasas (.82).

\section{Estadísticos descriptivos, análisis de fiabilidad y correlación bivariadas}

La estadística descriptiva, la fiabilidad de las escalas y las correlaciones, se presenta en la tabla 2. Los resultados muestran una fiabilidad de las escalas que oscilan entre Alpha $=.80$ y Alpha $=.91$, las cuales muestran las medias, desviaciones estándar y correlaciones entre los diferentes tipos de motivaciones, la intención de ser físicamente activo y la satisfacción intrínseca en el deporte. 
Tabla 2.

Media, desviación estándar, coeficiente Alpha y correlaciones bivariadas de todas las variables.

\begin{tabular}{lllllllllllllllll}
\hline$N^{*}$ & Variables & $\mathrm{M}$ & $\mathrm{DS}$ & $@$ & 1 & 2 & 3 & 4 & 5 & 6 & 7 & 8 & 9 & 10 & 11 & 12 \\
\hline 1 & MI General & 5.87 & 1.16 & .88 & - & $.89^{* *}$ & $.88^{* *}$ & $.87^{* *}$ & $.83^{* *}$ & $.85^{* *}$ & $.20^{* *}$ & .04 & .04 & $.71^{* *}$ & $.76^{* *}$ & $-.20^{* *}$ \\
2 & MI Conocimiento & 5.86 & 1.17 & .91 & - & - & $.90^{* *}$ & $.89^{* *}$ & $.87^{* *}$ & $.87^{* *}$ & $.24^{* *}$ & $.07^{*}$ & $.07^{*}$ & $.69^{* *}$ & $.75^{* *}$ & $-.19^{* *}$ \\
3 & MI Estimulación & 5.78 & 1.19 & .90 & - & - & - & $.90^{* *}$ & $.88^{* *}$ & $.88^{* *}$ & $.28^{* *}$ & $.09^{* *}$ & $.08^{* *}$ & $.70^{* *}$ & $.77^{* *}$ & $-.15^{* *}$ \\
4 & MI Consecución & 5.88 & 1.14 & .90 & - & - & - & - & $.87^{* *}$ & $.90^{* *}$ & $.25^{* *}$ & .05 & $.06^{*}$ & $.69^{* *}$ & $.74^{* *}$ & $-.18^{* *}$ \\
5 & R Integrada & 5.70 & 1.23 & .91 & - & - & - & - & - & $.87^{* *}$ & $.34^{* *}$ & $.15^{* *}$ & $.14^{* *}$ & $.71^{* *}$ & $.75^{* *}$ & $-.13^{* *}$ \\
6 & R Identificada & 5.94 & 1.08 & .89 & - & - & - & - & - & - & $.24^{* *}$ & .04 & .05 & $.71^{* *}$ & $.75^{* *}$ & $-.17^{* *}$ \\
7 & R Introyectada & 4.53 & 1.56 & .80 & - & - & - & - & - & - & - & $.76^{* *}$ & $.69^{* *}$ & $.28^{* *}$ & $.24^{* *}$ & $.38^{* *}$ \\
8 & R Externa & 3.98 & 1.76 & .85 & - & - & - & - & - & - & - & - & $.81^{* *}$ & $.15^{* *}$ & $.08^{* *}$ & $.53^{* *}$ \\
9 & Desmotivación & 4.20 & 1.71 & .86 & - & - & - & - & - & - & - & - & - & $.12^{* *}$ & $.07^{* *}$ & $.53^{* *}$ \\
10 & MIFA & 4.22 & .76 & .81 & - & - & - & - & - & - & - & - & - & - & $.78^{* *}$ & $-.12^{* *}$ \\
11 & Diversión & 4.24 & .79 & .88 & - & - & - & - & - & - & - & - & - & - & - & $-.19^{* *}$ \\
12 & Aburrimiento & 2.55 & 1.38 & .86 & - & - & - & - & - & - & - & - & - & - & - & - \\
\hline
\end{tabular}

Nota: M= Media, DS= Desviación Estándar, @ Alfa de Cronbach, Ml= Motivación Intrínseca, $\mathrm{R}=$ Regulación, MIFA= Medida de la Intencionalidad de ser Físicamente Activa. ${ }^{* *} p<.01 ;{ }^{*} p<.05$

Fuente: Programa estadístico SPSS v24.

Además, en la Tabla 2. se distingue que los estudiantes muestran una puntuación moderada alto en la motivación intrínseca de $(M=5.87)$, tanto hacia el conocimiento ( $M=5.86)$ como hacia la estimulación $(M=5.78)$ y a la consecución $(M=5.88)$, motivación extrínseca ( $M=5.70)$, tanto en el factor identificación $(M=5.94)$, siendo moderadas las puntuaciones de introyección $(M=4.53)$, en regulación externa $(M=3.98)$ y en desmotivación ( $\mathrm{M}=4.20)$, con una puntuación moderada tenemos a la intención de ser físicamente activo $(M=4.22)$, a la satisfacción intrínseca diversión $(M=4.24)$ y con una puntuación baja en aburrimiento (M=2.55). 
El análisis de correlaciones indican que la Motivación General se relacionó es positiva y significativa con la motivación intrínseca hacia el conocimiento, estimulación, consecución, las tres formas de motivación extrínseca (integrada, identificada, introyectada), con la Medida de Intencionalidad de ser Físicamente Activo, las mismas se relacionaron de manera positiva y significativa con la Satisfacción Intrínseca en el Deporte diversión, y de manera negativa y significativa para el aburrimiento.

Del mismo modo, la motivación intrínseca de conocimiento, de estimulación, de consecución, se relaciona de una manera positiva y significativa con todas las variables de estudio y de manera negativa y significativa para el aburrimiento. Por otra parte, la motivación extrínseca integrada, se relaciona de una manera positiva y significativa con todas las variables de estudio, de manera negativa y significativa para el aburrimiento. La regulación identificada se relaciona de una manera positiva y significativa con las demás variables de estudio a excepción con motivación intrínseca externa y desmotivación, de manera negativa y significativa para el aburrimiento. La regulación introyectada, externa, la Desmotivación, la Intención de ser físicamente activo y la Satisfacción Intrínseca / Diversión se relaciona de una manera positiva y significativa con todas las variables de estudio.

\section{DISCUSIÓN}

El presente estudio investigativo, tiene como objetivo comparar la influencia de la motivación en la intención de ser físicamente activo y satisfacción deportiva en Educación Física mediante estudio de correlaciones bivariadas que tienen una relación positiva y significativa en estudiantes de Educación Física de la Unidad Educativa Fiscomisional "Don Bosco" de Macas, además, determinar los niveles de las variables estudiadas y las dimensiones de estudio en función de la edad, género, grado o curso. Estos resultados son consistentes con varios estudios de (Cera et al., 2015) con estudiantes de EF entre 12 y 16 años de edad, conformada por 170 integrantes siendo 95 chicos y 75 chicas, de Educación Secundaria Obligatoria, ubicado en la provincia de 
Huelva, resultados que se correlacionan con nuestro estudio de una manera positiva y significativa entre la MI con la $R$ identificada, $R$ introyectada y de manera significante con la $R$ externa, y de manera negativa con la desmotivación.

Investigaciones como la de (Espinoza-Arias \& Heredia-León, 2021), aplicada a 403 estudiantes de EF las cuales 196 son hombres y 207 son mujeres, con edades comprendidas entre 9 a 18 años, presenta correlaciones entre las $\mathrm{Ml}$ en todos los niveles, de igual forma las motivaciones extrínsecas en todos sus ámbitos y la Intención de ser Físicamente Activo; lo cual se asemeja positivamente con los resultados obtenidos en este trabajo, siendo el aburrimiento significante pero de manera negativa.

Este estudio ha coincidido con investigaciones de (Usán \& Salavera, 2018) realizado con estudiantes entre los 12 y 18 años de edad, de 18 establecimientos de Educación Secundaria Obligatoria de la provincia de Zaragoza, donde analizan variables motivacionales que se relaciona positivamente con nuestro estudio siendo estas $\mathrm{Ml}$ de conocimiento, $\mathrm{R}$ Externa, $\mathrm{R}$ identificada, $\mathrm{R}$ introyectada y así mismo se relaciona negativamente con la Desmotivación.

En el ámbito deportivo la investigación de (Almagro et al., 2011), realizado con 580 atletas, 105 de sexo femenino y 475 de sexo masculino, entre los 12 y 17 años de edad, estas muestras fueron recogidas de escuelas deportivas municipales y clubes deportivos de diferentes localidades de una provincia española, se relaciona con este estudio de investigación que se consiguieron una relación positiva y significativa de $\mathrm{Ml}$ de conocimiento, de estimulación y de ejecución y la intención de ser físicamente activo.

Otro estudio, llevado por la investigación de (Freire, 2016), realizado por 99 deportistas tanto de femeninos como masculinos entre edades de 12 a 24 años que practican diferentes deportes en clubes de Buenos Aires, se corroboran estudios con el nuestra investigación con una correlación positiva y significativa con la MI de conocimiento, $\mathrm{Ml}$ de estimulación, además se relaciona con la motivación extrínseca de $R$ identificada, $R$ introyectada, $R$ externa y se relaciona también de manera negativa con la Desmotivación. Similar a nuestra investigación, en estudios anteriores elaborados en el contexto 
deportivo de (Balaguer et al., 2008), constituido por 301 deportistas, 171 hombres y 130 mujeres, que intervenían en diferentes deportes, preparándose más de cuatro días a la semana, en edades comprendidas entre una edad media de 24.1 , está relacionada positivamente de manera significante con la MI y la R identificada y de manera negativa con la $\mathrm{R}$ externa y la desmotivación.

Los resultados conseguidos en el estudio de (Díaz-Sozoranga et al., 2020) con una muestra organizada de 535 alumnos de distintos instituciones educativas que pertenecen al distrito de educación 11D02 Catamayo, Chaguarpamba, Olmedo, entre 12 y 20 años de edad, 275 eran alumnos y 260 alumnas, se correlacionan con las variables de esta investigación de manera positiva entre la intención de ser físicamente activa con las variables motivacionales.

Tal y como han expuesto otros trabajos de (Franco et al., 2016) que tiene una muestra compuesta por 587 alumnos de diferentes instituciones educativas de Madrid, entre los 14 y 18 años de edad, 276 eran chicos y 311 chicas, alumnos de Educación Secundaria Obligatoria de 1ero de Bachillerato, las cuales se relacionan positivamente con esta investigación entre la variable de la motivación intrínseca y como predictores de la intención físicamente activa para realizar AF.

En líneas generales de resultados obtenidos de (Moreno et al., 2012), está compuesto de 1008 alumnos de educación secundaria obligatoria (442 chicos y 566 chicas), entre los 15 y 17 años de edad, en esta investigación se aplicaron los distintos perfiles motivacionales prestando atención a la autodeterminación y la intención de ser físicamente activo, que se relaciona con esta investigación.

Con respecto a los resultados obtenidos sobre la intencionalidad de ser físicamente activos de (Munuera et al., 2018), comprendida por 591 estudiantes (281 hombres y 310 mujeres) entre los 12 y 17 años de edad, de diferentes colegios públicos de Educación Secundaria Obligatoria de Murcia, cuyos resultados se relacionan de manera positiva y similares a los obtenidos por esta investigación. 
De hecho, investigaciones previas han expuesto que la variedad deportiva puede ser un elemento concluyente de motivación y de la responsabilidad deportiva de (Almagro \& Conde, 2012), estudio conformado por 261 deportistas de básquet de distintos clubes de Andaluza, entre los 12 y 17 años de edad, con 177 deportistas masculinos y 84 deportistas femeninas, se relaciona con este estudio investigativo, en la intención de ser físicamente activo se relacionó positivamente con las demas variables de forma positiva y estadísticamente significativa de esta investigación.

Así mismo investigaciones relacionas en el deporte de (Franco et al., 2012), con una muestra estaba formada por 181 jugadores de baloncesto en formación, con edades comprendidas entre 11 y 16 años. La intención de ser físicamente activo se correlacionó de manera positiva con la competencia y el resto de las variables.

Los resultados del estudio (Valero-Valenzuela et., al., 2019), con una muestra que formada por 313 atletas que pertenecían a 15 clubes de la Federación Española de Atletismo, compuesto por 254 atletas (97 hombres y 157 mujeres) participaron en la competencia oficial de campo traviesa (de 2 a 4 kilómetros), con edades comprendidas entre los 10 y los 16 años $(M=12,81, S D=1,89)$ y frecuencia media de entrenamiento de 3-4 veces por semana, este estudio se relaciona con los perfiles motivacionales y la intensión de ser físicamente activo con esta investigación.

Existen otros estudios de (Baena-Extremera \& Granero-Gallegos, 2015) con una participación de 125 escolares (59 chicos y 66 chicas) de 4to. año de Educación Secundaria Obligatoria, entre 15 y 16 años de edad, que pertenecen al colegio de enseñanza pública de España, resultados que se se relacionan con la satisfacción/diversión en estudiantes de EF, de manera significativa positiva frente a la motivación intrínseca y en escalas bajas en cuanto al aburrimiento.

Como afirma (Granero-Gallegos et al., 2014) con la participaron un total de 758 escolares (347 hombres y 411 mujeres) de educación secundaria de Murcia, con un rango de edad comprendida entre 13 y 18 años, se relacionan los resultados con este trabajo, con la satisfacción / diversión obtuvieron valores altos que el aburrimiento, como también se 
relaciona de manera positiva con las variables de MI, ME y la correlación con el aburrimiento fue negativa.

En el estudio de (Zueck et al., 2020), participaron 208 estudiantes de sexto año de primaria, consideraron un grupo de 115 (93 mujeres y 115 hombres), de cuatro escuelas pertenecientes a dos zonas escolares de la ciudad de Chihuahua, que afirman una correlación entre la motivación intrínseca y la satisfacción / diversión con efectos positivos en las clases de EF, lo cual se asemeja positivamente con el presente trabajo sobre las variables estudiadas.

Así mismo en el aspecto deportivo los estudios de (Abraldes et al., 2016) con la participación de un total de 163 nadadores, desde la categoría cadete hasta sénior de diferentes clubes, los resultados encontrados se relacionan con esta investigación cuyos valores de diversión son más altos que el aburrimiento.

En el estudio de investigación de (Baños et al., 2019), conformada por 457 estudiantes de secundaria, de los cuales 247 son de sexo femenino y 210 de sexo masculino, los análisis de correlación revelaron relaciones positivas y significativas entre las variables de satisfacción de diversión con la EF, además indica que nuestro estudio el aburrimiento se relacionaba con la desmotivación de los jóvenes hacia la práctica deportiva y la poca importancia que le hallan a la EF.

Finalmente, los estudios de (Gutiérrez-García et al., 2020) con la participaron de 373 escolares que se identifican a deportes de conjunto e individuales, se preparan con equipos de algunos estados de México, edades que oscilan entre los 12 y 16 años con 204 chicos y 169 chicas. Coinciden con los resultados de este estudio donde la Ml esta relaciona con la satisfacción / disfrute y se asocia negativamente con el aburrimiento.

\section{CONCLUSIONES}

Se estableció la relación entre la Motivación, la Intención de ser Físicamente Activo y la Satisfacción Intrínseca en el Deporte en estudiantes de Educación Física. Se encontró que los docentes estarían impulsando la motivación en las clases, de la misma forma 
incentivando la intención de ser físicamente activo en estudiantes que se está reflejando la diversión en las clases.

Se demostró que los estudiantes encuestados en Educación Física han alcanzado niveles normales, lo que demuestra en las correlaciones positivas entre la motivación intrínseca con la estimulación, la intención de ser físicamente activo, la diversión entre otras.

Además, se recomienda como parte de esta investigación realizar más estudios de este tipo, experimentales con metodología longitudinal, aplicando estrategias para la motivación, la intención de ser físicamente activo y la satisfacción intrínseca en el deporte.

\section{FINANCIAMIENTO}

No monetario.

\section{AGRADECIMIENTO}

A la Universidad Católica de Cuenca; por todo el apoyo brindado en la motivación y desarrollo de esta investigación.

\section{REFERENCIAS CONSULTADAS}

Abraldes, J., Granero-Gallegos, A., Baena-Extremera, A., Gómez-López, M., \& Rodríguez-Suárez, N. (2016). Orientaciones de meta, satisfacción, creencias de éxito y clima motivacional en nadadores [Goal Orientations, Satisfaction, Success Beliefs, and Motivational Climate Swimmers]. Revista Internacional de Medicina y Ciencias de la Actividad Física y del Deporte, 16(63), 583-599. https://doi.org/10.15366/rimcafd2016.63.011 
Almagro, B., \& Conde, C. (2012). Factores motivacionales como predictores de la intención de ser físicamente activos en jóvenes jugadores de baloncesto. [Motivational factors as predictors of the intention to be physically active in young basketball players]. Cuadernos de Psicología del Deporte, 12(1), $1-4$.

Almagro, B., Moreno, J., \& Sáenz-López, P. (2012). Perfiles motivacionales de deportistas adolescentes españoles [Motivational profiles of Spanish adolescent athletes] Revista de Psicología del Deporte, 21(2), 223-231.

Almagro, B., Sáenz-López, P., González, D., \& Moreno, J. (2011). Clima motivacional percibido, necesidades psicológicas y motivación intrínseca como predictores del compromiso deportivo en adolescentes. [Perceived motivational climate, psychological needs and intrinsic motivation as predictors of sports commitment in adolescents]. Revista Internacional de Ciencias del Deporte, 7(25), 250-265. https://doi.org/10.5232/ricyde2011.02501

Baena-Extremera, A., \& Granero-Gallegos, A. (2014). Versión española del Sport Satisfaction Instrument (SSI)

adaptado al aprendizaje de la Educación Física bilingüe en inglés. [Spanish version of the Sport Satisfaction Instrument (SSI) adapted to the learning of bilingual Physical Education in English]. Porta Linguarum. Revista interdepartamental e interuniversitaria, 2(24), 63-76.

Baena-Extremera, A., \& Granero-Gallegos, A. (2015). Efectos de las actividades en la naturaleza en la predicción de la satisfacción de la Educación Física [Effects of activities in nature on the prediction of satisfaction with Physical Education]. Nuevas Tendencias en Educación Física, Deporte y Recreación, 28, 9-14. https://doi.org/10.47197/retos.v0i28.34816

Balaguer, I., Castillo, I., \& Duda, J. (2008). Apoyo a la autonomía, satisfacción de las necesidades, motivación y bienestar en deportistas de competición: Un análisis de la teoría de la autodeterminación. [Support for autonomy, satisfaction of needs, motivation and well/being in competitive athletes: An analysis of the theory of self-determination]. Revista de Psicología del Deporte., 17(1), 18. 
Balaguer, I., Guivernau, M., Duda, J., \& Crespo, M. (1997). Análisis de la validez de constructo y de la validez predictiva del cuestionario de clima motivacional percibido en el deporte (PCMSQ-2) con tenistas españoles de competición. [Analysis of the construct validity and predictive validity of the questionnaire on motivational climate perceived in sport (PCMSQ-2) with Spanish competitive tennis players]. Revista de Psicología del Deporte, 6(1), 41-58.

Baños, R., Marentes-Castillo, M., Zamarripa, J., Baena-Extremera, A., Ortiz-Camacho, M. del M., \& Duarte-Félix, H. (2019). Satisfacción, aburrimiento e importancia de la educación física en la intención de realizar actividad física extraescolar en adolescentes mexicanos: Satisfacción, aburrimiento e importancia de la educación física. [Satisfaction, boredom and importance of physical education in the intention to carry out extracurricular physical activity in Mexican adolescents: Satisfaction, boredom and importance of physical education]. Cuadernos de Psicología del Deporte, 19(3), 205-215. https://doi.org/10.6018/cpd.358461

Carrasco, H., Chirosa, L., Martín, I., Cajas, B., \& Reigal, R. (2015). Efectos de un programa extraescolar basado en juegos reducidos sobre la motivación y las necesidades psicológicas básicas en las clases de educación [Effects of a reduced game-based afterschool program on motivation and basic psychological needs in education classe s]. Revista Iberoamericana de Psicología del ejercicio y el deporte, 10(1), 23-31.

Castillo, I., Balaguer, I., \& Duda, J. (2002). Las perspectivas de meta de los adolescentes en el contexto deportivo. [The goal perspectives of adolescents in the sports context]. Psicothema, 14(2), 280-287.

Cera-Castillo, E., Almagro, B., Conde, C., \& Sáenz-López Buñuel, P. (2015). Inteligencia emocional y motivación en educación física en secundaria [Emotional intelligence and motivation in physical education in secon dary school]. Retos, 27(1), 8-13. http://hdl.handle.net/10637/7625

Charchaoui, I., Cachón, J., Chacón, F., \& Castro, R. (2017). Tipos de motivación para participar en las clases de educación física en la etapa de enseñanza secundaria obligatoria (E.S.O.) [Types of motivation to participate in physical education classes in compulsory secondary education (E.S.O.)]. Acción Motriz, Revista Científica digital, 18, 37-46. 
Revista Arbitrada Interdisciplinaria KOINONIA

Año VI. Vol VI. N4. Edición Especial: Educación III. 2021

Hecho el depósito de Ley: FA2016000010 ISSN: 2542-3088

FUNDACIÓN KOINONIA (F.K). Santa Ana de Coro. Venezuela.

Lorena Elisabeth Basurto-Ortiz; Diego Andrés Heredia-León

Deci, E., \& Ryan, R. (2008). Self-determination theory: A macrotheory of human motivation, development, and health. Canadian Psychology/Psychologie Canadienne, 49(3), 182-185.

Duda, J., \& Nicholls, J. (1992). Dimensions of achievement motivation in schoolwork and sport. - PsycNET. Journal of Educational Psychology, 84(3), 290/299.

Espinoza-Arias, B., \& Heredia-León, D. (2021). Motivación e intención deportiva en estudiantes de Educación Física en tiempos de pandemia [Motivation and sports intention in Physical Education students in times of pandemic]. Ciencia Latina Revista Científica Multidisciplinar, 5(5), 6941 6956. https://doi.org/10.37811/cl rcm.v5i5.823

Fernándes, D., \& Silveira, M. (2012). Evaluación de la motivación académica y la ansiedad escolar y posibles relaciones entre ellas. [Assessment of academic motivation and school anxiety and possible relationships between them]. Revista Psico-USF, 17(3), 447-455. https://doi.org/10.1590/S1413$\underline{82712012000300011}$

Franco, E., Coterón, J., \& Pérez-Tejero, J. (2016). Intención de ser físicamente activos entre estudiantes de EF: diferencias según la obligatoriedad de la enseñanza [Intention to be physically active among PE students: differences according to the compulsory nature of teaching]. REEFD. Revista española de Educación Física y Deportes, 12(414), 39-51.

Franco, E., Coterón, J., Martínez, H. A., \& Brito, J. (2017). Perfiles motivacionales en estudiantes de educación física de tres países y su relación con la actividad física. [Motivational profiles in physical education students from three countries and their relationship with physical activity]. Suma Psicológica, 24(1), 1-8.

Franco, E., Pérez-Tejero, J., \& Arrizabalaga, A. (2012). Motivación e intención de ser físicamente activo en jugadores de baloncesto en formacion. Diferencias en función de la competición. [Motivation and intention to be physically active in basketball players in training. Differences depending on the competition]. Cuadernos de Psicología del Deporte, 12(1), 23-26. 
Revista Arbitrada Interdisciplinaria KOINONIA

Año VI. Vol VI. N4. Edición Especial: Educación III. 2021

Hecho el depósito de Ley: FA2016000010 ISSN: 2542-3088

FUNDACIÓN KOINONIA (F.K). Santa Ana de Coro. Venezuela.

Lorena Elisabeth Basurto-Ortiz; Diego Andrés Heredia-León

Freire, C. (2016). Motivación deportiva y clima motivacional percibido como predictores del compromiso deportivo en adolescentes deportistas del con urbano bonaerense [Sports motivation and motivational climate perceived as predictors of sports commitment in adolescent athletes from the Buenos Aires suburbs]. Recuperado desde https://repositorio.uade.edu.ar/xmlui/handle/123456789/4076

Garcés, T., Castro, M., Zurita, F., \& Martínez, A. (2015). Actividad física en escolares de educación primaria [Physical activity in primary school students]. Andalucía, 2(2), 223-228.

González-Cutre, D., Sicilia, Á., \& Moreno-Murcia, J. (2008). Modelo cognitivo-social de la motivación de logro en educación física [Cognitive-social model of achievement motivation in physical education]. Psicothema, 20(4), 642-651.

Granero-Gallegos, A., Baena-Extremera, A., Sánchez-Fuentes, J., \& Martínez-Molina, M. (2014). Perfiles motivacionales de apoyo a la autonomía, autodeterminación, satisfacción, importancia de la educación física e intención de práctica física en tiempo libre. [Motivational profiles to support autonomy, self-determination, satisfaction, importance of physical education and intention of physical practice in free time]. Cuadernos de Psicología del Deporte, 14(2), 59-70.

Gutiérrez-García, P., Castillo-Jiménez, N., Ríos-Domínguez, R., González-Fiambres, R., Ramírez-Siqueiros, M., \& López-Walle, J. (2020). Clima de empoderamiento, tipos de motivación, diversión y aburrimiento en jóvenes deportistas [Climate of empowerment, types of motivation, fun and boredom in young athletes]. Journal of Behavior, Health \& Social Issues, 12(2), 23-32.

Lim, C., \& Wang, J. (2009). Perceived autonomy support, behavioural regulations in physical education and physical activity intention. Psychology of Sport and Exercise, 10(1), 52-60. https://doi.org/10.1016/i.psychsport.2008.06.003

Lonsdale, C., Hodge, K., \& Rose, E. A. (2008). The Behavioral Regulation in Sport Questionnaire (BRSQ): Instrument Development and Initial Validity Evidence. Journal of Sport and Exercise Psychology, 30(3), 323-355. https://doi.org/10.1123/jsep.30.3.323 
Manzano-Sánchez,

D.,

$\&$

Valero-Valenzuela, A. (2013). Análisis del perfil motivacional de diversos grupos de atletas y su repercusión en el nivel de satisfacción deportiva. [Analysis of the motivational profile of various groups of athletes and its impact on the level of sports satisfaction]. SPORT TK-Revista EuroAmericana de Ciencias del Deporte, 2(2), 9-19. https://doi.org/10.6018/194571

Mendelsohn, D. (2001). La motivación en el fútbol. [Motivation in football]. Revista Digital, 7(37), 10.

Méndez-Giménez, A., Cecchini-Estrada, J., \& Fernández-Río, J. (2012). Autodeterminación y metas sociales: Un modelo estructural para comprender la intención de práctica, el esfuerzo y el aburrimiento en Educación Física [SelfDetermination and

Social Goals:

A Structural Model for Understanding Practice Intent, Effort, and Boredom in Physical Education]. Aula abierta, 40(1), 51-62.

Méndez-Giménez, A., Fernández-Río, J., \& Cecchini-Estrada, J. (2013). Climas motivacionales, necesidades, motivación y resultados en Educación Física. [Motivational climates, needs, motivation and results in Physical Educatio n]. Aula abierta, 41(1), 63-72.

Menéndez, J., \& Fernández-Río, J. (2017). Responsabilidad social, necesidades psicológicas básicas, motivación intrínseca y metas de amistad en educación

física [Social responsibility, basic psychological needs, intrinsic motivation and fri endship goals in physical education]. Retos Nuevas Tendencias en Educación Física, Deporte $\quad$ y $\quad$ Recreación, 32, 134-139. https://doi.org/10.47197/retos.v0i32.52385

Montero, I., \& León, O. (2007). A guide for naming research studies in Psychology. International Journal of Clinical and Health Psychology, 7(3), 847862.

Moreno, J., Cervelló, E., \& González-Cutre, D. G.-C. (2007). Analizando la motivación en el deporte: Un estudio a través de la teoría de la autodeterminación. [Analyzing motivation in sport: A study through the theory of self-determination]. APUNTES DE PSICOLOGÍA, 25(1), 35-51. 
Revista Arbitrada Interdisciplinaria KOINONIA

Año VI. Vol VI. N4. Edición Especial: Educación III. 2021

Hecho el depósito de Ley: FA2016000010 ISSN: 2542-3088

FUNDACIÓN KOINONIA (F.K). Santa Ana de Coro. Venezuela.

Lorena Elisabeth Basurto-Ortiz; Diego Andrés Heredia-León

Moreno, J., López de San Román, M., Martínez, C., Alonso, N., \& González-Cutre, D. (2008). Peers' Influence on Exercise Enjoyment: A Self-Determination Theory Approach. Journal of Sports Science \& Medicine, 7(1), 23-31.

Moreno, J., Martínez-Galindo, C., Moreno, V., Marcos-Pardo, P., Conte, L., \& Moreno, R. (2012). Motivación, Creencias de habilidad e intención de ser físicamente activo al finalizar la educación obligatoria. [Motivation, Beliefs of ability and intention to be physically active at the end of compulsory education]. Revista Mexicana de Psicología, 29(2), 175-183.

Moreno, J., Zomeño, T., Marín, L., Cervello, E., \& Ruiz, L. (2009). Variables motivacionales relacionadas con la práctica deportiva extraescolar en estudiantes adolescentes de educación física. [Motivational variables related to extracurricular sports practice in adolescent physical education students]. Apuntes. Educación física y deportes, 1(95), 38-43.

Moreno-Murcia, J., Huéscar-Hernández, E., \& Ruíz-González, L. (2019). Capacidad predictiva del apoyo a la autonomía en clases de educación física sobre el ejercicio

físico [Predictive ability to support autonomy in physical education classes on phy sical exercise]. Revista Latinoamericana de Psicología, 51(1), 30-37. https://doi.org/10.14349/rlp.2019.v51.n1.4

Moreno-Murcia, J., Sicilia, A., Saénz-López, P., González-Cutre, D., Almagro, B., \& Conde, C. (2014). Análisis motivacional comparativo en tres contextos de actividad física. [Comparative motivational analysis in three contexts of physical activity]. Revista Internacional de Medicina y Ciencias de la Actividad Física y del Deporte, 14(56), 665-685.

Munuera, J., Gómez-López, M., Granero-Gallegos, A., \& Sánchez-Alcaraz, B. (2018). Miedo al fallo de los estudiantes y la relación con la intención de ser físicamente activo e importancia de la Educación Física en Educación Secundaria [Fear of student failure and the relationship with the intention to be physically active and the importance of Physical Education in Secondary Education]. Estudios pedagógicos (Valdivia), 44(1), 279-291. 
Revista Arbitrada Interdisciplinaria KOINONIA

Año VI. Vol VI. N4. Edición Especial: Educación III. 2021

Hecho el depósito de Ley: FA2016000010

ISSN: 2542-3088

FUNDACIÓN KOINONIA (F.K). Santa Ana de Coro. Venezuela.

Lorena Elisabeth Basurto-Ortiz; Diego Andrés Heredia-León

Naranjo, M. (2009). Motivación: Perspectivas teóricas y algunas consideraciones de su importancia en el ámbito educativo [Motivation: Theoretical perspectives and some considerations of its im portance in the educational field]. Revista https://doi.org/10.15517/revedu.v33i2.510

$\begin{array}{llll}\text { Organización Mundial de la Salud. (2020). Actividad } & \end{array}$ física. [Physical activity]. Recuperado desde https://n9.cl/hkni

Rodríguez, M. (2009). Motivar para aprender en situaciones académicas (La crisis de la escuela educadora). [Motivate to learn in academic situations (The crisis of the educating school)]. Editorial Laertes. Recuperado desde https://n9.cl/gzyuo

Ryan, R., \& Deci, E. (2000). La Teoría de la Autodeterminación y la Facilitación de la Motivación Intrínseca, el Desarrollo Social, y el Bienestar. [The Theory of SelfDetermination and the Facilitation of Intrinsic Motivation, Social Development, and Well-being]. American Psychologist, 55(1), 16.

Sánchez-Oliva, D., Alonso, D., González-Ponce, I., García-Calvo, T., \& Leo, F. (2012). Desarrollo de un cuestionario para valorar la motivación en Educación Física [Development of a questionnaire to assess motivation in Physical Educatio n]. Revista Iberoamericana de Psicología del Ejercicio y el Deporte, 7(2),227-250. https://www.redalyc.org/pdf/3111/311126611010.pdf

Shephard, R., \& Trudeau, F. (2000). The Legacy of Physical Education: Influences on Adult Lifestyle. Pediatric Exercise Science, 12(1), 3450. https://doi.org/10.1123/pes.12.1.34

Sproule, J., Wang, J., Morgan, K., McNeill, M., \& McMorris, T. (2007). Effects of motivational climate in Singaporean physical education lessons on intrinsic motivation and physical activity intention. Personality and Individual Differences, 43(5), 1037-1049. https://doi.org/10.1016/j.paid.2007.02.017

Usán, P., \& Salavera, C. (2018). Motivación escolar, inteligencia emocional y rendimiento académico en estudiantes de educación secundaria obligatoria. [School motivation, emotional intelligence and academic performance in compulsory secondary education students]. Actualidades en Psicología, 32(125), 95-112. https://doi.org/10.15517/ap.v32i125.32123 
Valero-Valenzuela, A., Manzano-Sánchez, D, Moreno-Murcia \& Heredia-León, D. (2019). Interpersonal Style of Coaching, Motivational Profiles and the Intention to be Physically Active in Young Athletes. Studia Psychologica, 61(2), 110-119. https://doi.org/10.21909/sp.2019.02.776

Zueck, M. del C., Ramírez, A. A., Rodríguez, J. M., \& Irigoyen, H. E. (2020). Satisfacción en las clases de Educación Física y la intencionalidad de ser activo en niños del nivel de primaria. [Satisfaction in Physical Education classes and the intention to be active in elementary school children]. Retos: nuevas tendencias en educación física, deporte y recreación, 37, 33-40 